\title{
CORRESPONDING GROUP AND MODULE SEQUENCES ${ }^{11}$
}

\author{
R. H. CROWELL
}

\section{$\S 1$. Statement of results}

For convenience we consider throughout an arbitrary but fixed multiplicative group $H$. The integral group ring of $H$ is denoted by $Z H$, and the homomorphism $\varepsilon: Z H \rightarrow Z$ is always the trivializer, or unit augmentation, defined by $\varepsilon h=1$ for all $h \in H$. For any group extension of $H$, i.e., exact sequence of multiplicative groups

$$
1 \longrightarrow K \longrightarrow G \stackrel{\varphi}{\longrightarrow} H \rightarrow 1
$$

we shall construct an exact sequence of left $\mathrm{ZH}$-modules

$$
0 \longrightarrow B \longrightarrow A \stackrel{\partial}{\longrightarrow} Z H \stackrel{\varepsilon}{\rightarrow} Z \longrightarrow 0 .
$$

(All unlabeled monomorphisms will be assumed to be inclusion mappings). We shall say that the module sequence (2) is determined by the group sequence (1).

Conversely, starting from an arbitrary exact sequence (2) of left $Z H$-modules, we shall construct a multiplicative group $\bar{A}$ and an abelian group extension

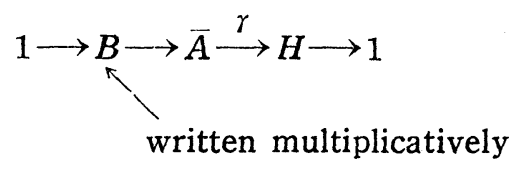

whose kernel is the additive group of the module $B$. We shall also say that the group sequence (3) is determined by the module sequence (2).

Two group extensions (1) will be called equivalent if there exists an isomorphism of one onto the other which is the identity on $H$. Similarly, two module sequences (2) are equivalent if there exist a $Z H$-isomorphism of one onto the other which is the identity on $Z H$. It will be obvious that equivalent group sequences determine equivalent module sequences and conversely.

Received January 23, 1961.

1) This work was supported by a grant (G-8931) from the National Science Foundation. 
Where $K^{\prime}$ is the commutator subgroup of $K$, a group sequence (1) induces the commutative diagram

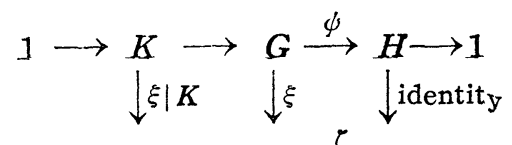

$$
\begin{aligned}
& 1 \longrightarrow K / K^{\prime} \longrightarrow G / K^{\prime} \stackrel{\zeta}{\rightarrow} H \longrightarrow 1
\end{aligned}
$$

We shall call the second row of the diagram, which is also exact, the abelianization of the first. The principal theorems of this paper are

(1.1) The group sequence determined by the module sequence determined by a given group sequence (1) is equivalent to the abelianization of (1).

And, conversely,

(1.2) The module sequence determined by the group sequence determined by a given module sequence (2) is equivalent to (2).

It is an immediate corollary that the module sequences determined by two group sequences are equivalent if and only if their abelianizations are equivalent.

The module sequence determined by a group sequence (1) will be shown in $\S 6$ to be the 0 - and 1-dimensional part of the homology sequence of the pair $(G, 1)$ with coefficients in $Z H$ as defined by Massey [?]. If $G$ is given by a group presentation $\left(x_{1}, x_{2}, \ldots: r_{1}, r_{2}, \ldots\right)_{p}$, the module sequence (2) may be calculated using the tree differential culus of $\overline{F o x}[4,5]$. The matrix $\left\|\phi \varphi\left(\frac{\partial r_{i}}{\partial x_{j}}\right)\right\|$ is a relation matrix for the relative homology group $H_{1}(G, 1 ; Z H)$, which is the module $A$ in the sequence (2). These facts and (1.1) imply Blanchfield's result [1] that, to quote Fox, "roughly speaking, the Jacobian class of $G$ at $\psi$ determines the structure of $G$ modulo the commutator subgroup of the kernel of $\psi^{\prime \prime}$. Even though the approach and proofs are entirely different, many of Blanchfield's basic constructions translate directly into the ones used here. In $\S 7$ I have elaborated on the comparison.

I am greatly indebted to Hale $F$. Trotter of Queer's University, Kingston, Ontario, with whom I was associated in the NSF project ${ }^{1}$. His suggestions have resulted in substantial simplifications in the present treatment.

\section{§2. Construction of the module sequence}

Consider given a group extension (1). Let $\psi: Z G \rightarrow Z H$ be the linear ex- 
tension to the group rings of the group homomorphism $\psi$, and let $\varepsilon: Z G \rightarrow Z$ be the trivializer of $G$. The ideals that are the kernels of $\phi$ and $\varepsilon$ we denote by $\mathscr{A}$ and $\mathbb{S}$, respectively. Where $\mathscr{R} S$ is the product ideal and $\pi$ is the factor homomorphism, we obtain the commutative diagram

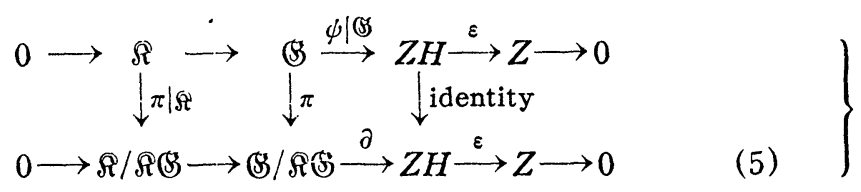

whose rows are easily seen to be exact. Observe that $\$ / \Re(S$ is a left $Z H$ module with the operation of $Z H$ well-defined by

$$
\psi u \cdot \pi v=\pi(u v), \text { for all } u \in Z G \text { and } v \in \mathbb{B} .
$$

Furthermore, $\mathscr{R} / \mathscr{R}$ is a submodule and the mappings in the second row (5) are $Z H$-homomorphisms. The sequence (5) is by definition the module sequence (2) determined by (1).

\section{$\S 3$. Construction of the group extension}

Consider an arbitrary exact module sequence (2) (the mapping $\varepsilon$ is always the trivializer). With respect to a product defined by

$$
a b=a+(\partial a+1) \cdot b, \text { for all } a, b \in A,
$$

the module $A$ is a semi-group with the element 0 the identity.

Let $\bar{A}$ be the set of all $a \in A$ such that $\partial a+1 \in H$. Then,

(3.1) A is a multiplicative group (the inverse of $a$ is $a^{-1}=-(\partial a+1)^{-1} \cdot a$ ).

Notice that $B \subset \bar{A}$ and, in addition,

$$
b_{1}+b_{2}=b_{1} b_{2}, \quad \text { for any } b_{1}, b_{2} \in B .
$$

Thus $B$ is a subgroup of $\bar{A}$. We define the mapping $\gamma: \ddot{A} \rightarrow H$ by setting $r a=\partial a+1$, all $a \in \bar{A}$. It is straightforward to show that

\section{(3.3) $\gamma$ is a group homomorphism of $\bar{A}$ onto $H$ with kernel $B$.}

We therefore obtain the abelian extension (3). In any abelian extension there is defined an operation of the image on the kernel. In particular, the action of $H$ on $B$ is well defined by

$$
r a \circ b=a b a^{-1}, \text { for any } a \in \bar{A} \text { and } b \in B .
$$


This product can be extended in the obvious way so that $B$ is a $Z H$-module with respect to $\circ$. Inasmuch as

$$
\gamma a \circ b=a b\left(-(\gamma a)^{-1} \cdot a\right)=a\left(b-(\gamma a)^{-1} \cdot a\right)=a+\gamma a \cdot b-a=\gamma a \cdot b,
$$

it follows that

(3.4) The induced module structure on $B$ is the same as the original.

\section{§. The principal mapping diagram}

Consider a given group sequence (1), and let (5) be the module sequence which it determines. The principal mapping diagram is the commutive diagram

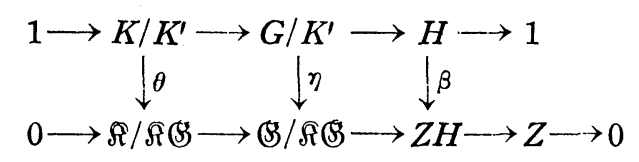

The mapping $\beta$ is defined by $\beta h=h-1$, for all $h \in H$. Let $\alpha: G \rightarrow \mathbb{B}$ be given by $\alpha g=g-1$, for all $g \in G$. The mapping $\eta$ is defined by the commutativity relation

$$
\eta \xi=\pi \alpha
$$

(The diagram chasing in the following lemmas is based on (4) and (6).)

(4.1) $\eta$ is well-defined.

Proof. Observe first of all that $\alpha$ is a crossed homomorphism :

$$
\alpha\left(g_{1} g_{2}\right)=g_{1} g_{2}-1=\left(g_{1}-1\right)+g_{1}\left(g_{2}-1\right)=\alpha g_{1}+g_{1} \alpha g_{2} .
$$

Hence, for any $k_{1}, k_{2} \in K$,

$$
\alpha k_{1} k_{2} k_{1}^{-1} k_{2}^{-1}=\left(1-k_{1} k_{2} k_{1}^{-1}\right) \alpha k_{1}+k_{1}\left(1-k_{2} k_{1}^{-1} k_{2}^{-1}\right) \alpha k_{2} .
$$

Thus, by (2.1), $\pi \alpha k_{1} k_{2} k_{1}^{-1} k_{2}^{-1}=0$; whence it follows that $\pi \alpha K^{\prime}=0$. Next, suppose that $\xi g_{1}=\xi g_{2}$. Then, $g_{1} g_{2}^{-1} \in K^{\prime}$ and so

$$
0=\pi \alpha g_{1} g_{2}^{-1}=\pi\left(\alpha g_{1}-g_{1} g_{2}^{-1} \alpha g_{2}\right)=\pi \alpha g_{1}-\pi \alpha g_{2},
$$

and the proof is complete.

(4.2) $\partial \eta=\beta \zeta$.

Proof. For any $g \in G, \quad \partial \eta \xi g=\partial \pi \alpha g=\psi(g-1)$ and $\beta \zeta \xi g=\beta \psi g=\psi g-1$. 
(4.3) For any $r_{1}, r_{2} \in G / K^{\prime}, \eta\left(r_{1} \boldsymbol{r}_{2}\right)=\eta r_{1}+\zeta r_{1} \cdot \eta r_{2}$, i.e., $\eta$ is a crossed homomorphism.

Proof. Choose counter-images $r_{1}=\xi g_{1}$ and $r_{2}=\xi g_{2}$. Then,

$$
\begin{aligned}
\eta\left(r_{1} r_{2}\right) & =\eta \xi g_{1} g_{2}=\pi \alpha g_{1} g_{2}=\pi\left(\alpha g_{1}+g_{1} \alpha g_{2}\right) \\
& =\pi \alpha g_{1}+\psi g_{1} \cdot \pi \alpha g_{2}=\eta \xi g_{1}+\zeta \xi g_{1} \cdot \eta \xi g_{2} \\
& =\eta r_{1}+\zeta r_{1} \cdot \eta r_{2} .
\end{aligned}
$$

Inasmuch as $\partial \eta r=\beta \zeta r=1-1=0$ for any $r \in K / K^{\prime}$, it follows that the image of the restriction $\theta=\eta \mid K / K^{\prime}$ is contained in $\mathscr{R} / \mathscr{N} \mathbb{S}$. Thus the existence of the principal mapping diagram (7) is established. It is a consequence of (4.3) that

$$
\theta\left(r_{1} r_{2}\right)=\theta r_{1}+\theta r_{2}, \quad \text { for any } r_{1}, r_{2} \in K / K^{\prime} .
$$

Hence, $\theta$ is a multiplicative-to-additive group homomorphism. In addition $\theta$ is a $Z H$-homomorphism. The operation of $H$ on $K / K^{\prime}$ is defined by $h \cdot r=s r s^{-1}$, ior all $h \in H, r \in K / K^{\prime}$, and $s$ such that $\zeta s=h$ (just like the definition of $\circ$ in the paragraph preceding $(3.4)$ ). We have

$$
\begin{aligned}
\theta(h \cdot r) & =\eta\left(s r s^{-1}\right) \\
& =\eta s+\zeta s \cdot \eta r-\zeta\left(s r s^{-1}\right) \cdot \eta s \\
& =h \cdot \theta r,
\end{aligned}
$$

which is sufficient to verify that $\theta$ is a $Z H$-homomorphism.

\section{(4.4) The mapping $\theta$ is a ZH-isomorphism onto.}

Proof. The proof is obtained by constructing the inverse mapping. Choose a system of coset representatives, i.e., for each $h \in H$, select $\vec{h} \in G$ such that $\psi \bar{h}=h$, and consider an arbitrary element $\sum_{g \in G} n_{g} g$ in the ideal $\Re$. Then

$$
\begin{gathered}
\sum_{h \in G} n_{g} g=\sum_{h \in H} \sum_{k \in K} n_{\bar{h} k} \bar{h} k \\
0=\psi\left(\sum_{g \in G} n_{g} g\right)=\sum_{h \in H}\left(\sum_{k \in K} n_{\bar{h} k}\right) h
\end{gathered}
$$

Since $H$ is a basis for the free additive group $Z H, 0=\sum_{k \in K} n \overline{h k}$. Hence,

$$
\sum_{g \in G} n_{g} g=\sum_{h \in H} \sum_{k \in K} n \bar{h} k \bar{h}(k-1) .
$$

In addition, if $\sum_{h, k \neq 1} n_{\bar{h} k} \bar{h}(k-1)=0$, then all $n_{\bar{h} k}=0$. It follows that the elements 
$\bar{h}(k-1)$, for all $h \in H$ and $1 \neq k \in K$, constitute a basis for the free additive group $\Re$. We may therefore define an additive-to-multiplicative homomorphism v : $\rightarrow K / K^{\prime}$ by

$$
v(\bar{h}(k-1))=h \cdot \xi k, \quad h \in H \text { and } k \in K .
$$

Consider arbitrary elements $g \in G$ and $k \in K$. Where $h=\phi g$, we have $g=\bar{h} k^{\prime}$ for some $k^{\prime} \in K$. Then,

$$
g(k-1)=\bar{h}\left(k^{\prime} k-1\right)-\bar{h}\left(k^{\prime}-1\right),
$$

and so

$$
v(g(k-1))=\left(h \cdot \xi k^{\prime} k\right)\left(h \cdot \xi k^{\prime}\right)^{-1}=\psi g \cdot \xi k .
$$

This implies

$$
v(u v)=\psi u \cdot \nu v, \quad \text { for all } u \in Z G \text { and } v \in \Re .
$$

Consider the identity

$$
(k-1)(g-1)=g\left(g^{-1} k g-1\right)-(k-1), \quad g \in G \text { and } k \in K .
$$

Inasmuch as $\zeta \xi g=\psi g$, we have, according to the definition of the operation of $H$ on $K / K^{\prime}$,

$$
v\left(g\left(g^{-1} k g-1\right)\right)=\psi g \cdot \xi\left(g^{-1} k g\right)=\xi\left(g g^{-1} k g g^{-1}\right)=\xi k .
$$

Hence,

$$
v(k-1)(g-1)=(\xi k)(\xi k)^{-1}=1 .
$$

The ideal $\mathscr{R} S$ is a left $Z G$-module generated by all elements $(k-1)(g-1)$, $k \in K$ and $g \in G$. As a result of the above equation and (4.5), we conclude that $v\left(\mathscr{A}(\mathbb{S})=1\right.$. Consequently, $v$ induces a homomorphism $\omega: \Re / \mathscr{H} \rightarrow K / K^{\prime}$, which is easily seen by (4.5) and (2.1) to be $Z H$-linear. Since $\xi K$ and $\{\pi(k-1)\}_{k \in K}$ generate the modules $K / K^{\prime}$ and $\mathscr{R} / \mathfrak{K} \mathbb{S}$, respectively, the equations

$$
\begin{aligned}
\theta \xi k & =\pi(k-1) \\
\omega \pi(k-1) & =v(k-1)=\xi k
\end{aligned}
$$

show that $\omega \theta=$ identity and $\theta \omega=$ identity, and the proof is complete.

The preceding result, (4.4), is the key lemma of this paper. I had. originally proved it using the free calculus of Fox, specifically, $\$ 4$ of his paper [4]. Later K. Iwasawa suggested a proof independent of group presentations. The present proof was constructed in a discussion with Trotter and was motivated 
by an analogous argument on page 190 of [2]. It is a corollary of (4.4) and the principal mapping diagram that if $k \in K$ and $k-1 \in \Re\left(\right.$, then $k \in K^{\prime}$. This is a result proved by Fox for the case that $G$ is a free group, cf. (4.9) in [4]. Fox's paper also contains references to earlier proofs by Schuman and Blanchfield.

\section{$\S 5$. Proofs of $(1.1)$ and $(1.2)$}

The proof of (1.1) is an immediate corollary of (4.4) and the principal mapping diagram. We write the second row of the latter as $0 \rightarrow B \rightarrow A \stackrel{\partial}{\rightarrow} Z H$ $\stackrel{\varepsilon}{\rightarrow} Z \rightarrow 0$, and consider the abelian extension $0 \rightarrow B \rightarrow \bar{A} \stackrel{\gamma}{\rightarrow} H \rightarrow I$ which it deter. mines. Diagram chasing around (7) yields

(5.1) $\bar{A}=$ image $\eta$ and $\gamma \eta=\zeta$.

(5.2) $\eta$ is a group isomorphism of $G / K^{\prime}$ onto $\bar{A}$.

Proof. That $\eta$ is a homomorphism follows from

$$
\begin{aligned}
\eta\left(r_{1} r_{2}\right) & =\eta r_{1}+\zeta r_{1} \cdot \eta r_{2} \\
& =\eta r_{1}+\gamma \eta r_{1} \cdot \eta r_{2}=\left(\eta r_{1}\right)\left(\eta r_{2}\right) .
\end{aligned}
$$

Next suppose that $\eta r=$ identity for some $r \in G / K^{\prime}$. Since the identity element of $\bar{A}$ is 0 , we have

$$
0=\partial \eta r=\beta \zeta r=\zeta r-1
$$

It follows that $r \in K / K^{\prime}$ and thence that $0=\eta r=\theta r$. Since, by (4.4), $\theta$ is a multiplicative to additive isomorphism, we conclude that $r=1$. This completes the proof.

As a result, the commutative diagram

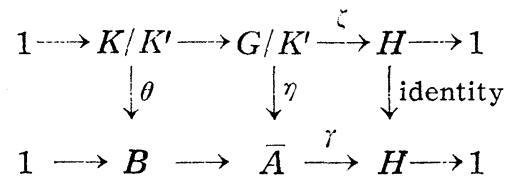

exhibits the equivalence of the two rows, and theorem (1.1) is proved.

To prove (1.2), we start with an arbitrary module sequence (2). In order to avoid the necessity of introducing new notation in what follows, we write the group sequence (3) determined by (2) as $1 \rightarrow K \rightarrow G \stackrel{\xi}{\rightarrow} H \rightarrow 1$. This group 
extension in turn detemines the module sequence (5), and it is our objective to show that (2) and (5) are equivalent. The additive group of $Z G$ is freely generated by $G$. Since $G=\bar{A} \subset A$, the inclusion can be extended to an additive group homomorphism $i: Z G \rightarrow A$.

(5.3) $i(u v)=(\psi u) \cdot(i v)$, for any $u \in Z G$ and $v \in \mathbb{B}$.

Proof. It is sufficient to prove that $i(g v)=(\psi g) \cdot(i v)$ for any $g \in G$ and $v \in \mathbb{B}$. Since $v=\sum_{g \in G} n_{g}(g-1)$, it is actually enough to show

$$
i\left(g_{1}\left(g_{2}-1\right)\right)=\left(\phi g_{1}\right) \cdot i\left(g_{2}-1\right), \quad g_{1}, g_{2} \in G .
$$

The identity $1 \in G$ is the element $0 \in A$, Thus $i 1=0$. Furthermore, $\psi=\gamma$, and so according to the definition of multiplication in the semi-group $A$, the equation $g_{1} g_{2}=g_{1}+\psi g_{1} \cdot g_{2}$ holds in $A$. Therefore,

$$
\begin{aligned}
i\left(g_{1}\left(g_{2}-1\right)\right) & =i\left(g_{1} g_{2}-g_{1}\right)=g_{1} g_{2}-g_{1} \\
& =\psi g_{1} \cdot g_{2}=\left(\psi g_{1}\right) \circ i\left(g_{2}-1\right),
\end{aligned}
$$

and this completes the argument.

(5.4) $\quad i(\Re(3)=0$.

Proof. In view of (5.3), it suffices to verify the assertion on a generator $(k-1)(g-1), k \in K$ and $g \in G$.

$$
\begin{aligned}
i(k-1)(g-1) & =i(k g-k-g+1) \\
& =k g-k-g=k+\psi k \cdot g-k-g=0 .
\end{aligned}
$$

As a result, there exists the following commutative diagram of additive group homomorphisms

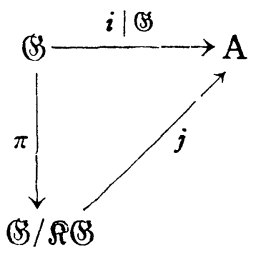

(5.5) $j$ is a ZH-homomorphism.

Proof. This amounts to checking

$$
j(\psi u \cdot \pi v)=\psi u \cdot j \pi v, \quad \text { for any } u \in Z G \text { and } v \in \mathbb{G} .
$$


By (2.1), (5.3), and the above diagram

$$
j(\phi u \cdot \pi v)=j \pi u v=i u v=\phi u \cdot i v=\psi u \cdot j \pi v .
$$

Since in the present instance $B=K$, the commutator subgroup $K^{\prime}$ is trivial. This simplifies the fundamental mapping diagram, and we obtain $\eta: G \rightarrow \mathbb{S} / \mathfrak{A}(S$. For any $g \in G$, we have $\eta g=\pi(g-1)$, and so $j \eta g=j \pi(g-1)=i(g-1)=g$. That is,

(5.6) The composition $G^{-} \stackrel{\eta}{\rightarrow}\left(\mathbb{S} / \Re\left(S^{j} \rightarrow A\right.\right.$ is the inclusion mapping.

Inasmuch as $\eta \mid K$ is the isomorphism $\theta$, it follows from (5.6) that $j$ maps $\Re / \mathscr{R} \&$ onto $K=B$ and that the restriction of $j$ to $\mathscr{R} / \mathscr{R} S$ is the inverse $\theta^{-1}$. We therefore obtain the diagram

$$
\begin{aligned}
& 0 \longrightarrow \Re / \mathscr{R} \rightarrow\left(\Re / \Re \& \stackrel{\partial_{1}}{\rightarrow} Z H \stackrel{\varepsilon}{\longrightarrow} Z \longrightarrow 0\right. \\
& \downarrow \theta^{-1} \quad \downarrow j \quad \downarrow \text { identity } \downarrow \text { identity } \\
& 0 \longrightarrow B \longrightarrow A \stackrel{\hat{o}_{2}}{\longrightarrow} Z H \stackrel{\varepsilon}{\longrightarrow} Z \longrightarrow 0
\end{aligned}
$$

whose rows are the module sequences (5) and (2). To establish commutativity, it remains to show

(5.7) $\partial_{2} j=\partial_{1}$.

Proof. We need $\partial_{2} j_{\pi} v=\partial_{1} \pi v$, for any $v \in \mathbb{B}$; so it suffices to prove $\partial_{2} j \pi(g-1)=\partial_{1} \pi(g-1)$, for any $g \in G$. The mapping $\gamma=\psi$ is defined by $\partial_{2} g$ $+1=\gamma g=\psi g . \quad$ By $(5), \partial_{1} \pi(g-1)=\psi g-1$. Hence, $\partial_{2} j \pi(g-1)=\partial_{2} i(g-1)$ $=\partial_{2} g=\psi g-1=\partial_{1} \pi(g-1)$, and the proof is complete.

Thus (8) is a commutative diagram. Since $\theta^{-1}$ is an isomorphism, it follows from the "five" lemma that $j$ is also, We conclude that the rows are equivalent, and theorem (1.2) is proved. It is interesting that according to (5.6) the mapping $j^{-1}$ is an extension of $\eta$ from $G=\bar{A}$ to $A$. If we could produce this extension directly, (1.2) would be an immediate corollary of the principal mapping diagram.

\section{§. The homology sequence}

In this section we show that the module sequence (5) determined by a given group extension (1) is the 0 - and 1-dimensional part of the homology sequence of the pair $(G, 1)$ with coefficients in $Z H$. 
For any ring homomorphism $\psi: A \rightarrow \Lambda^{\prime}$ (a ring is assumed to have an identity) and right $A^{\prime}$-module $B^{\prime}$, we define the right $A$-module $B_{\psi}^{\prime}$ whose underlying additive group is the same as that of $B^{\prime}$ and whose scalar multiplication is defined by $b^{\prime} \cdot \lambda=b^{\prime} \cdot \psi \lambda$. For any left $A$-module $A$, we define the left $A^{\prime}$-module ${ }_{(\psi)} A=\Lambda_{\psi}^{\prime} \otimes_{\Lambda} A$. The mapping $\dot{\psi}_{A}: A \rightarrow{ }_{(\psi)} A$ defined by $\psi_{A}(a)=1^{\prime} \otimes_{\Lambda} a$ obviously satisfied

$$
\psi_{A}(\lambda \cdot a)=\psi \lambda \cdot \psi_{A}(a), \quad \text { for all } \lambda \in \Lambda \text { and } a \in A .
$$

Assuming that $\psi$ is onto and using the right exactness of the tensor product, we obtain from the exact sequence $0 \rightarrow \operatorname{kernel} \stackrel{i}{\rightarrow} \stackrel{i}{\rightarrow} \stackrel{\dot{\varphi}}{\rightarrow} \Lambda_{\psi}^{\prime} \rightarrow 0$ of right $A$-modules the commutative diagram

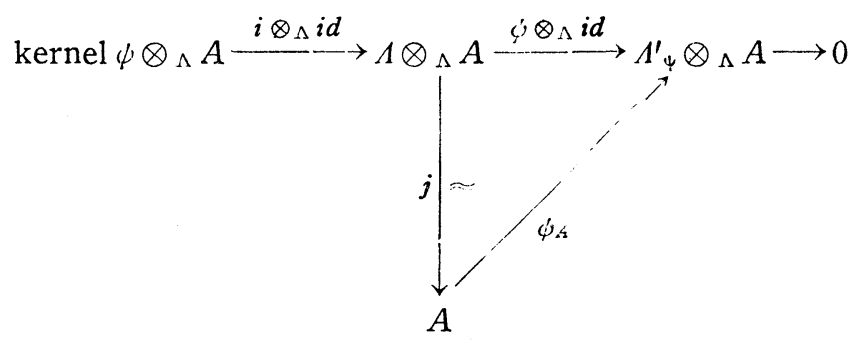

whose row is exact. The isomorphism $j$ is the mapping $\lambda \otimes_{\Lambda} a \rightarrow \lambda \cdot a$, and the image of kernel $\psi \otimes_{\Lambda} A$ under $j\left(i \otimes_{\Lambda} i d\right.$ ) is (kernel $\left.\psi\right) \cdot A$. We conclude that

(6.1) If $\psi$ is onto, then $0 \rightarrow(\operatorname{kernel} \psi) \cdot A \rightarrow A \stackrel{\dot{\varphi}_{A}}{\longrightarrow}(\psi) A \rightarrow 0$ is exact.

Finally, we remark that any $A$-homomorphism $f: A_{1} \rightarrow A_{2}$ of two left $A^{-}$ modules induces a $1^{\prime}$-homomorphism $f^{\prime}:{ }_{(\varphi)} A_{1} \rightarrow(\varphi) A_{2}$ in the obvious way and that $\psi_{A_{2}} f=f^{\prime} \psi_{A_{1}}$.

Our construction of the homology sequence of $(G, 1)$ follows Trotter's formulation [8] for a group system. The system here consists simply of the group $G$, the trivial subgroup $\{1\}$, and the inclusion $\{1\} \rightarrow G$. The module of coefficients is $Z H_{\psi}$, where $\psi: Z G \rightarrow Z H$ is the extension to the group rings of the group homomorphism $\psi$. Let $X$ be a projective $Z G$-resolution of $Z$,

$$
\longrightarrow X_{2} \stackrel{d_{2}}{\rightarrow} X_{1} \stackrel{d_{1}}{\rightarrow} Z G \stackrel{\varepsilon}{\longrightarrow} Z \longrightarrow 0 .
$$

The subcomplex $Y$ corresponding to $\{1\}$ is

$$
\rightarrow 0 \rightarrow 0 \longrightarrow Z G \stackrel{\varepsilon}{\rightarrow} Z \longrightarrow 0
$$


The graded structure of the chain mapping $\psi_{X}: X \rightarrow(\psi) X$ is exhibited in

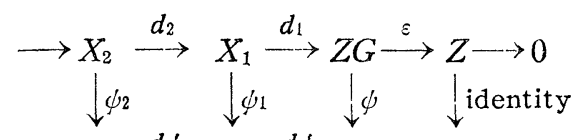

$$
\begin{aligned}
& \rightarrow(\psi) X_{2}{\stackrel{d_{2}^{\prime}}{\longrightarrow}}_{(\psi)} X_{1} \stackrel{d_{1}^{\prime}}{\longrightarrow} Z H \stackrel{\varepsilon}{\longrightarrow} Z \longrightarrow 0
\end{aligned}
$$

For $n \geq 1$, we have abbreviated $\psi_{x_{n}}$ by $\psi_{n}$. The module $Z H_{\psi} \otimes{ }_{G} Z G$ has been identified with $Z H$ and, therefore, $\phi_{Z G}$ is replaced by $\psi$. The homology sequence of $(G, 1)$ with coefficients in $Z H$ is that of the pair $\left(_{(\psi)} X,(\psi) Y\right)$. That is, of

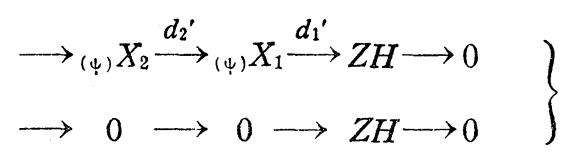

It follows that the 0- and 1-dimensional part of this sequence is of the form $0 \rightarrow H_{1}(G ; Z H) \rightarrow H_{1}(G, 1 ; Z H) \stackrel{\partial}{\rightarrow} Z H \stackrel{\varepsilon}{\longrightarrow} Z \rightarrow 0$. In order to establish its equivalence with $(5)$, it will suffice to establish a commutative diagram

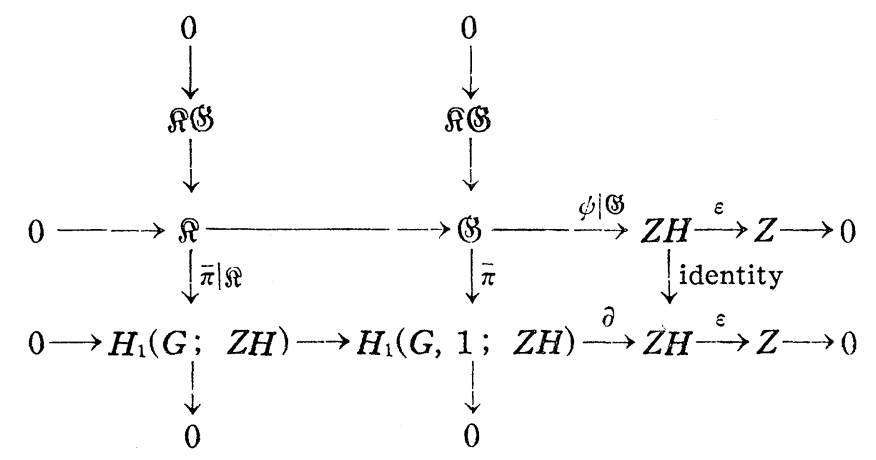

whose rows and columus are exact.

From the exact sequence $\rightarrow X_{2} \stackrel{d_{2}}{\longrightarrow} X_{1} \stackrel{e}{\rightarrow} \& \rightarrow 0$. where $e x=d_{1} x$, for all $x \in X_{1}$, we obtain the commutative diagram

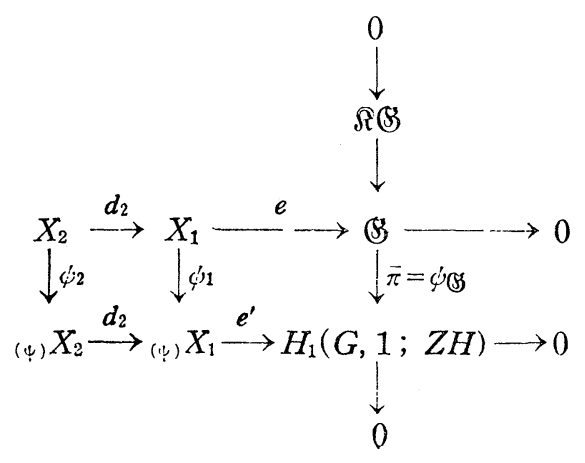


The second row is exact by the right exactness of the tensor product and the column is exact by (6.1).

The remaining two lemmas serve to complete the proof of the existence of the mapping diagram (9).

(6.2) $\bar{\pi} \Re=H_{1}(G ; Z H)$.

Proof. An element of $H_{1}(G, 1 ; Z H)$ belongs to $H_{1}(G ; Z H)$ if and only if it has a counter-image under $e^{\prime}$ in kernel $d_{1}^{\prime}$. Consider $v \in \mathscr{R}$ and select $x \in X_{1}$ such that $e x=d_{1} x=v$. Then $\bar{\pi} v=e^{\prime} \psi_{1} x$ and $d_{1}^{\prime} \psi_{1} x=\psi d_{1} x=\psi v=0$. Hence $\pi v \in H_{1}(G ; Z H)$. Conversely, consider $b \in H_{1}(G ; Z H)$. Select $x^{\prime} \in$ kernel $d_{1}^{\prime}$ such that $e^{\prime} x^{\prime}=b$, and choose $x \in X_{1}$ such that $\psi_{1} x=x^{\prime}$. Since $\phi e x=\psi d_{1} x$ $=d_{1}^{\prime} \psi_{1} x=d_{1}^{\prime} x^{\prime}=0$, we have $e x \in \Re$. Since $\bar{\pi} e x=e^{\prime} \psi_{1} x=e^{\prime} x^{\prime}=b$, we conclude $b \in \pi \Re$. This completes the proof.

\section{(6.3) $\partial \bar{\pi}=(\phi \mid(\$)$.}

Proof. It follows from the definition of the boundary operator in an homology sequence that $\partial \boldsymbol{e}^{\prime}=d_{1}^{\prime}$. Hence,

$$
\partial \bar{\pi} e=\partial e^{\prime} \psi_{1}=d_{1}^{\prime} \psi_{1}=\psi d_{1}=(\psi \mid \mathbb{B}) e .
$$

Since $e$ is onto, the proof is complete.

\section{§7. Jacobian matrices and Blanchfield's senior thesis}

Suppose $G$ is defined by a group presentation $G=\left(x_{1} x_{2}, \ldots: r_{1}, r_{2}, \ldots\right)_{p}$. That is, there is given a free group $F$ freely generated by $x_{1}, x_{2}, \ldots$ and a homomorphism $\varphi$ of $F$ onto $G$ with kernel the consequences of $r_{1}, r_{2}, \ldots$ (cf. $[4,5])$. We first show that the Jacobian matrix at $\psi$, i.e., $\psi \varphi\left(\frac{\partial \boldsymbol{r}_{i}}{\partial x_{j}}\right)$, is a relation matrix for $H_{1}(G, 1 ; Z H)$. Following Trotter, we define the complex

$X_{0}=Z G$

$X_{1}=$ free $Z G$-module generated by $a_{j} \leftrightarrow x_{j}, j=1,2, \ldots$

$X_{2}=$ free $Z G$-module generated by $b_{i} \leftrightarrow r_{i}, i=1,2, \ldots$

$d_{1}: X_{1} \rightarrow X_{0}$ defined by $d_{1} a_{j}=\varphi x_{j}-1, j=1,2, \ldots$

$d_{2}: X_{2} \rightarrow X_{1}$ defined by $d_{2} b_{i}=\sum_{j} c\left(\frac{\partial r_{i}}{\partial x_{j}}\right) \cdot a_{j}, i=1,2, \ldots$

It is not hard to prove that the sequence $X_{2} \stackrel{d_{2}}{\rightarrow} X_{1} \stackrel{d_{1}}{\longrightarrow} X_{0} \stackrel{\varepsilon}{\longrightarrow} Z \rightarrow 0$ is exact (cf. lemma 5.1 of $[6])$. It is therefore the 2 -skeleton of a projective $Z G$-resolution 
of $Z$. Consider the mapping diagram (10) in which $X_{1}$ and $X_{2}$ are assumed to be as described in this section. The $Z H$-modules $(\psi) X_{1}$ and ${ }_{(\psi)} X_{2}$ are free and generated by $\left\{\psi_{1} a_{1}, \psi_{1} a_{2}, \ldots\right\}$ and $\left\{\psi_{2} b_{1}, \psi_{2} b_{2}, \ldots\right\}$, respectively. Since

$$
d_{2}^{\prime} \psi_{2} b_{l}=\psi_{1} d_{2} b_{i}=\sum_{j} \psi \varphi\left(\frac{\partial r_{i}}{\partial x_{j}}\right) \cdot \psi_{1} a_{j}, \quad i=1,2, \ldots
$$

it follows that $\mid \psi \varphi\left(\begin{array}{c}\partial r_{i} \\ \partial x_{j}\end{array}\right)$ is the matrix of $d_{2}^{\prime}$ and therefore a relation matrix of $H_{1}(G, 1 ; Z H)$.

Blanchfield proved that, for any finite presentation $\left(x_{1}, \ldots, x_{n}: r_{1}, \ldots\right.$, $\left.r_{m}\right)_{p}=G$, the matrix $P=\psi \varphi\left(\frac{\partial r_{i}}{\partial x_{j}}\right), i=1, \ldots, m, j=1, \ldots, n$, and the column matrix $Q=\left\|\psi \varphi x_{j}-1\right\|, j=1, \ldots, n$, determine the groups $K / K^{\prime}$ and $G / K^{\prime}$. In view of the preceding paragraph, this result follows at once from our theorem (1.2). We have seen that specifying $P$ is the same as specifying $d_{2}^{\prime}$. Similarly, $Q$ defines $d_{1}^{\prime}$ inasmuch as

$$
d_{1}^{\prime} \psi_{1} a_{j}=\psi d_{1} a_{j}=\psi \varphi x_{j}-1 .
$$

Finally, the sequence

$$
\text { (ب) } X_{2} \stackrel{d_{2}^{\prime}}{\longrightarrow}(\varphi) X_{1} \stackrel{d_{1}^{\prime}}{\longrightarrow} Z(H) \stackrel{\varepsilon}{\rightarrow} Z \longrightarrow 0
$$

defines the homology sequence (2) which, by (1.1), determines

$$
1 \rightarrow K / K^{\prime} \rightarrow G / K^{\prime} \rightarrow H \rightarrow 1 .
$$

Blanchfield proved (4.4) for finitely generated groups. That is, he has

$$
K / K^{\prime} \approx \text { kernel } d_{1}^{\prime} / \text { image } d_{2}^{\prime},
$$

where $d_{1}^{\prime}$ and $d_{2}^{\prime}$ are defined by the matrices $Q$ and $P$. Interestingly enough, he did not consider $H_{1}(G, 1 ; Z H)=A$ but a group which can be identified as the image of $G / K^{\prime}$ under $\eta$, i.e., our group $A$.

\section{BIBLIOGRAPH Y}

[1] Blanchfield, R. C., Applications of free differential calculus to the theory of groups, Senior thesis, Princeton University, 1949.

[2] Cartan, H., and Eilenberg, S., Homological Algebra, Princeton University Press, 1956.

[3] Eilenberg, S., and MacLane, S., Cohomology theory in abstract groups, Ann. of Math., Vol. 48 (1947), pp. 51-78.

[4] Fox, R. H., Free differential calculus I, Ann. of Math., Vol. 57 (1954), pp. 547-560.

[5] Fox, R. H., Free differential calculus II, Ann. of Math., Vol. 59 (1954), pp. 136-210. 
[6] Lyndon, R. C., Cohomology groups with a single defining relation, Ann. of Math., Vol. 52 (1950), pp. 650-665.

[7] Massey, W. S., Some problems in algebraic topology and the theory of fibre bundles, Ann. of Math., Vol. 62 (1955), pp. 327-357.

[8] Trotter, H. F., Homology of group systems with applications to knot theory, forthcoming.

Dartmouth College 